Folatos y riesgo de cáncer de mama

\section{Folate and breast cancer risk}

\section{Sr Editor:}

Recientemente revisé el artículo de Castillo et al. "Folatos y riesgo de cáncer de mama: revisión sistemática" publicado en la Revista ${ }^{1}$. Tengo varios comentarios sobre este artículo, incluyendo los datos utilizados por los autores para llegar a sus conclusiones.

Primero, la ingesta de folato fue definido al pie de la Tabla 2 como "folato dietario; ácido fólico como suplemento vitamínico; y folato total (folato dietario + ácido fólico contenido en suplementos vitamínicos)". Tanto en los métodos como en los resultados, no se especifica cómo se definió la ingesta de ácido fólico de alimentos fortificados.

La Figura 2 presenta el OR relacionando folato plasmático con el riesgo de cáncer de mama, de estudios caso-control anidado. Todos los datos graficados incluyen 1 en el intervalo de confianza, lo cual sugiere que no hay relación estadísticamente significativa entre folato plasmático y cáncer de mama.

La Figura 3 presenta el RR relacionando ingesta dietaria de folato y cáncer de mama en estudios de cohorte. La mayoría de los estudios en la Figura 3 no muestran asociación entre la ingesta dietaria de folato y el riesgo de cáncer de mama, y dos de los estudios muestran un efecto protector de la ingesta de folato en el riesgo de cáncer de mama.

La Figura 4 muestra el RR relacionando ingesta total de folato y riesgo de cáncer de mama en estudios de cohorte. La mayoría de los estudios en la Figura 4 no muestran una asociación entre la ingesta total de folato y el riesgo de cáncer de mama, y un estudio muestra un efecto protector de la ingesta total de folato y el riesgo de cáncer de mama.

No se presentan los datos de los estudios caso-control (no anidados) y estudios clínicos aleatorios, listados en la Tabla 2. Por lo tanto, no se puede concluir si existe o no una relación entre folato y cáncer de mama en estos estudios.
Al final de la Tabla 2 se presentan resultados de dos metaanálisis. Todos los OR y RR incluyen 1 en el intervalo de confianza, lo que indica que no hay relación estadísticamente significativa entre folato y riesgo de cáncer de mama. No se aclara en la Tabla si para el estudio de Lewis 2006 los datos son bioquímicos o dietéticos.

En resumen, la mayoría de la evidencia graficada en las Figuras 2 a 4, y presentada en la Tabla 2, indica que no hay relación entre folato (plasmático, dietaria o dietaria total) y riesgo de cáncer de mama, o un efecto protector entre folato y riesgo de cáncer de mama. Además, ningún análisis evaluó directamente si la ingesta de ácido fólico proveniente de alimentos fortificados tenía relación con el riesgo de cáncer de mama.

Por estas razones, considero que las conclusiones de los autores no están apoyadas por los datos presentados en el artículo: "The results support the need to be cautious and to limit the exposure of women to high intakes of folic acid, especially in countries with mandatory food fortification."

Helena Pachón, PhD, MPH

Senior Nutrition Scientist, Flour Fortification Initiative. Research Associate Professor, Hubert Department of Global Health. Emory University.

\section{Reference}

1. Castillo C, Tur JA, Uauy R. Folatos y riesgo de cáncer de mama: revisión sistemática. Rev Med Chile 2012; 140: 251-60.

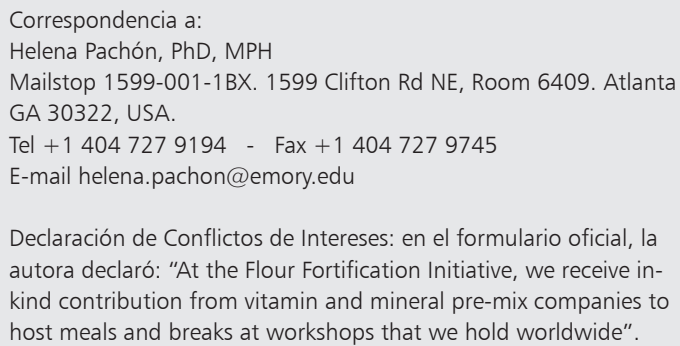
autora declaró: "At the Flour Fortification Initiative, we receive inkind contribution from vitamin and mineral pre-mix companies to host meals and breaks at workshops that we hold worldwide". 